\title{
Mobile health applications in rheumatology: Could they improve our care and research?
}

With $>165,000$ mobile health apps available on iTunes and Google Play mHealth is a rapidly growing field, which generated high expectations for the improvement of our health care. We identify two uses for mHealth in the field of rheumatology: A) aiding patient care and B) the systematic collection of data for scientific research. Currently, there is still a lack of evidence that mHealth will live up to the high promises. In this review we discuss the pros and cons of mHealth and the research that is needed to develop and validate $\mathrm{mHealth}$ to ensure high quality apps.

Keywords: $\mathrm{mHealth} \cdot$ applications • evidence-based medicine $•$ self-management $•$ data collection

\section{Introduction}

The influence of mobile Health (mHealth), defined by the WHO as the "medical and public health practice supported by mobile devices such as mobile phones, patient monitoring devices, Personal Digital Assistants (PDAs), and other wireless devices" [1], is rapidly expanding. The number of mobile applications (apps) aimed at improving healthcare has increased throughout recent years, with $>165,000$ mobile health apps available on iTunes and Google Play [2-5]. The various app marketplaces showcase a plethora of different apps with the majority of the apps concentrated in the areas of wellness, diet and exercise and a quarter of them focusing on disease and treatment management for chronic diseases [5]. Amongst them there is an increasing number of apps aiming at our rheumatology patient population; By 2017, there were $>350$ arthritis applications on the Google Play store and $>100$ on the iTunes store [6].

Within mHealth apps we can distinguish seven functionality dimensions [5]:

- Inform: Provide information in a variety of formats (text, photo, video).

- Instruct: Provide instructions to the user.

- Record: Capture user entered data.

- Display: Graphically display/output user entered data.

- Guide: Provide guidance based on user entered information, and may further offer a diagnosis, or recommend a consultation with a physician or a treatment.

\author{
Maurits MP' ${ }^{1}$, Yuminaga $\mathbf{H}^{1}$, \\ Huizinga TWJ' \& Knevel $R^{* 1,2,3}$ \\ 'Department of Rheumatology, Leiden \\ University Medical Centre, Leiden, The \\ Netherlands \\ ${ }^{2}$ Division of Rheumatology, Brigham \\ and Women's Hospital, Harvard Medical \\ School, Boston, USA \\ ${ }^{3}$ Broad institute, Cambridge, USA \\ *Author for correspondence: r.knevel@ \\ lumc.nl
}

- Remind/Alert: Provide reminders to the user.

- Communicate: Provide communication between healthcare providers and patients.

This variety in functionalities creates high expectations for mHealth. The European Union states that mHealth "improves the empowerment of patients: they could manage their health more actively, live more independently thanks to selfassessment or remote monitoring solutions" and that it can "support healthcare professionals in treating patients more efficiently as mobile apps can encourage adherence to a healthy lifestyle" [7]. Others are less enthusiastic, stating that the field of mHealth "... is currently in its infancy and can be likened to the snake oil salesmen of the 1860s" (Steve Flat, director of the Psychological Therapies Unit in Liverpool) [8].

To us the functionalities demonstrate potential, but also spur our interest in the scientific foundation of this growing field, which we will discuss in this brief review in the light of the rheumatology clinic. We distinguished two important uses for mHealth apps:

- The aiding of patient care by increasing patient self-management via improving access to general disease information, collection of personal disease information and encouragement to improve one's behavior.

- The systematic collection of data for scientific research which could increase our understanding of differences between patients, possible disease triggers and 
Table 1. Theoretical pros and cons of mHealth applications.

\begin{tabular}{|c|c|}
\hline Pros & Cons \\
\hline \multicolumn{2}{|l|}{ mHealth for patient care } \\
\hline $\begin{array}{l}\text { Instruct: Improved self-management } \\
\text { - Rx and visit reminders } \\
\text { - lifestyle information and support } \\
\text { - more insight in one's own health (e.g. early flare recognition, } \\
\text { insight into positive behavioural effects) } \\
\text { Inform: Better informed about health care options } \\
\text { Peer support through social patient platform } \\
\text { Support clinical communication } \\
\text { - improving communication with health care workers } \\
\text { - Inter visit disease activity recall }\end{array}$ & $\begin{array}{l}\text { Becoming obsessive with self-control } \\
\text { - Unwelcome reminders of disease } \\
\text { - Incorrect sense of control over disease by simple } \\
\text { life-style interventions } \\
\text { - Over interpretation of data } \\
\text { - Increased healthcare consumption through } \\
\text { incorrect signals } \\
\text { Risk of communication of unfunded information }\end{array}$ \\
\hline \multicolumn{2}{|l|}{ mHealth for research purpose } \\
\hline $\begin{array}{l}\text { Data collection outside clinics } \\
\text { - symptom pattern } \\
\text { - comorbidities } \\
\text { - concomitant symptoms } \\
\text { - disease triggers } \\
\text { - additional measurements via gadgets } \\
\text { Overcome recall bias }\end{array}$ & Selection bias \\
\hline \multicolumn{2}{|l|}{ General } \\
\hline High prevalence of smartphones & $\begin{array}{l}\text { Privacy issues } \\
\text { Free-market instruments are not obliged to adhere } \\
\text { to medical standards of quality }\end{array}$ \\
\hline
\end{tabular}

concomitant symptoms (Table 1). We will address these two uses separately.

\section{Literature Review \\ mHealth for patient care}

\section{Self-management through instruction}

From a patient perspective the use of mobile apps can be very appealing, as it offers a way to influence their own health, in other words, their self-management. Studies showed that patients with chronic illnesses actively seek information online and desire to self-manage using smartphone applications [1].

Possibly the most basic feature of a patientoriented healthcare application is automatic reminders. By alerting the patient of pending or missed medication doses for example, treatment adherence could be substantially improved [9-11], which would subsequently lead to a better disease prognosis $[12,13]$. Notifications could also be incorporated for upcoming face-to-face visits, reducing the number of missed appointments and thus improving both treatment and healthcare efficiency [14]. Secondly, apps help patients self-manage their diseases whenever and wherever they choose [15]. Patients can register their general wellbeing, track disease symptoms such as joint pain and swelling, fatigue, morning stiffness, fever etc. which may lead to earlier recognition of disease activity and the timely seeking of help. Thirdly, apps can encourage people to improve their life-style by giving advice, self-evaluate and self-monitor their physical activity, eating habits, sleep behavior etc. This allows patients to recognize triggers for unhealthy behavior.

For the above-mentioned features to be advantageous, apps should meet certain criteria: advice should be correct, data should be easily interpretable and ideally information is evidence based and data is collected according to existing and validated guidelines. Unfortunately, these conditions are not always met. Grainger et al. evaluated app adherence to recommendations for monitoring of Rheumatoid Arthritis (RA) disease activity in clinical practice. From the 19 identified apps, only 8 included an ACR and EULAR-recommended RA composite disease activity measure [6]. Another large review examined studies of (general) medical apps that assessed the quality of these apps by evaluating expert involvement or adherence of app content to medical evidence [16]. In the 28 studies on expert involvement (concerning 3,852 apps), $9 \%-67 \%$ of the apps involved experts. Thirty studies evaluated the adherence to medical evidence in 3,051 apps. Seventeen of those studies found that none of the assessed apps $(n=2,237)$ adhered fully to the compared 
evidence. In the 13 other studies $10 \%-87 \%$ of the apps adhered fully to evidence [16].

Lack of medical evidence can make apps ineffective and discourage patients to improve their health. Unfortunately, apps can even be harmful; apps suggesting medication doses to patients through opioid-conversion without scientific validation [16] inaccurately calculate insulin dose for diabetes care [17] or incorrectly draws diagnoses [5].

Besides the question of scientific validity of the apps, the question arises how exactly patients' behavior is influenced by mHealth. First of all, is it effective? The lack of health behavior theory [18] integration suggests a high chance for lack of efficacy [19]. More seriously, studies have found that apps can lead to obsessive behavior resulting in deterioration of the users' health [20].

\section{Peer support through social platforms}

The main function of smartphones is connecting people with their peers. A healthcare app can facilitate this by acting as a platform for patients to meet and talk about their experiences. Diseases such as RA can severely impact the mental health of those afflicted [21]. Online patient-patient support groups could reduce this impact, thus improving quality of life for a group in sore need thereof. A patient oriented market study would be able to shed light on whether this would find a following, currently these have been limited to very small sample sizes [22-25].

\section{Inform}

Apps can improve self-care by informing patients about therapy options and new research findings, enablingshared decision-making between patients and their rheumatologists, which has been linked to improvement of prognosis [26]. Importantly, apps should also inform patients about the difference in evidence from one observational study versus meta-analyses or clinical trials. Next, it should be clear which findings concern general health and which concern their rheumatic disease. Finally, information is ideally tailored to the local situation and legislation (e.g. on drug prescription) of patients' residence to avoid patients feeling undertreated by their rheumatologist.

\section{Support clinical communication}

In addition to shared decision-making, apps can facilitate communication about disease activity and physical complaints. Patients are regularly asked how their disease activity has been since the last visit, often as much as half a year ago [27]. A task which can be hard to perform and inevitably leads to recall bias. Recording selfassessed disease activity using a smartphone app has been shown to aid in this inter-visit symptom recall $[28,29]$. It would be interesting to examine how this additional information influences rheumatologists' treatment decisions and patients' wellbeing.

While initial use of healthcare apps would most likely be supplemental to standard clinical care, legislators are anticipating a more substitutive role [7]. By connecting patient and clinician, the applications could reduce face-to-face visits, and thereby the time impact of routine checkups [30]. This has been proven helpful in fields where certain patient groups are hard to reach such as mental health care [31] and nutritional advice [32]. However, not all patients will be equally pleased about this development; those less enthused by the mobile application might see a reduction of consultations as diminishing care [30]. Physicians have expressed concern over the lack of non-verbal cues in written data, indicating less physical interactions could also result in missed information [33]. On the other hand, apps might even increase health care consumption by encouraging patients to seek help.

\section{mHealth for research purpose}

An interesting aspect of mHealth is the possibility of large-scale data collection through the construction of comprehensive databanks for epidemiological studies, but also to support clinical trials by reaching out to patients via notifications etc. The IMS institute for healthcare informatics wrote an extensive report in 2015 on the adoption of mHealth [5]. They found 300 clinical trials that utilize mHealth apps on the clinicaltrials.gov website. Of these trials $89 \%$ were interventional, targeting treatment and prevention, and the remainders were observational [5]. Interestingly, such trials had a $60 \%$ higher inclusion rate than conventional studies [34].

\section{Data collection}

Data collected through mHealth could have several benefits over conventional methods: it provides a denser overview of patients' wellbeing and disease activity, as well as of large-scale disease trends [35] and apps can take additional measurements by incorporating mobile phone gadgets. Studies have successfully used both 
physical extensions to the device such as a dynamometer, built-in hardware in the form of internal gyroscope's and accelerometers and digital functionality such as Global Positioning Systems, in order to measure extra variables ranging from patient grip strength to gait parameters to the local weather in order to correlate these to predetermined outcomes, with varying degrees of success [36-38]. The additional data on disease patterns, such as involvement of particular joints, comorbidities and triggers, which might go unnoticed in clinics, together with the potential to supplement such data with more novel information acquired via these peripheral methods, could prove invaluable. Whether these possible pros outweigh the as-ofyet insufficiently researched cons, remains to be investigated.

\section{Bias}

Though mHealth could help overcome recall bias, it can induce selection bias. In an era where there is still a clear, albeit fading, divide between generations in techno-literacy one can expect to find an artificially lowered average age in a group of smartphone using patients [39-42]. Similarly, level and type of education correlates to people's tendency to participate in any form of study, a phenomenon which is unlikely influenced by the change of medium, and which will therefore contribute further to a skewing of patient inclusion [40]. Furthermore, socioeconomically less developed people are likely underrepresented due to the high cost associated with acquiring and owning a smartphone [42]. This is particularly problematic because people with low socioeconomic background are already associated with higher computer illiteracy and poorer health due to their high stress levels, weaker social support group, lack of knowledge about diseases, and poorer coping styles $[29,36]$. Studies that found correlations between electronic PatientReported Outcome Measures (e-PROMs) and such variables as treatment adherence and disease coping could well be influenced by this bias $[11,43]$.

Still, this does not mean that the people missed by mHealth-based-studies are more likely to participate in the paper-based traditional studies. Regardless, researchers should strive to recruit a wide variety of patients and develop a study that would benefit the entire patient population.

\section{Conclusion}

mHealth is expanding rapidly and there is an enormous amount of health apps available. mHealth could help overcome current patient and scientific problems such as lack of information, coping difficulties, treatment adherence etc. Though these promises are hopeful, studies have repeatedly shown that the quality of apps varies widely, as many apps lack scientific evidence or experts' input. Some apps (outside of the field of rheumatology) have proven to actually be harmful. Even when apps are based on scientific studies and built with input from experts, they tend to lack evidence that they achieve what they aim for.

Ideally, mHealth is based on guidelines created by experts based on scientific evidence, such as the ACR and EULAR guidelines. In addition, involvement of behavioural scientist could improve the effectiveness of apps and prevent harm. Next, similar to other health interventions such as drugs, physiotherapy or life-style advice, $\mathrm{mHealth}$ necessitates scientific evaluation in order to recognise the effectiveness and (unforeseen) side effects. Finally, (thus far neglected in this review) privacy should be guaranteed.

The European union is funding programs for research and innovation on the development of innovative mHealth solutions via Horizon 2020 and the European commission has published a legal framework for lifestyle and wellbeing apps, which provides legislation for app developers [44]. In our opinion, ACR and EULAR have a great infrastructure due to the possibility to initiate taskforces. Such taskforces could formulate guidelines for app development and assessment, which could serve as quality standards for patients, clinicians, and scientists to select and develop apps. Via such initiatives, we are not only facing an era with exponential technological options, but also an era where these technological options improve the quality of our care and research.

\section{References}

1. WHO. mHealth: New Horizons for Health Through Mobile Technologies. Global Observatory for eHealth Series. (2011).

2. Silva BM, Rodrigues JJ, de la Torre Díez I et al. Mobile-health: a review of current state in 2015. J. Biomed. Inform. 56, 265-272 (2015).

3. Boulos MN, Brewer AC, Karimkhani C et al. Mobile medical and health apps: state of the art, concerns, regulatory control and certification. Online. J. Public. Health. Inform. 5(3), 229 (2014).

4. Aitken M, Gauntlett C. Patient apps for improved 
healthcare: from novelty to mainstream. IMS Institute for Healthcare Informatics. (2013).

5. Aitken M, Gauntlett C. Patient Adoption of mHealth Use, Evidence and Remaining Barriers to Mainstream Acceptance. IMS institute for healthcare Bioinformatics. (2015)

6. Grainger R, Townsley H, White B et al. Apps for people with rheumatoid arthritis to monitor their disease activity: a review of apps for best practice and quality. JMIR. Mhealth. Uhealth. 5(2), e7 (2017).

7. https://ec.europa.eu/digital-single-market/en/ mhealth

8. https://www.theguardian.com/science/2017/ feb/21/health-apps-could-be-doing-more-harmthan-good-warn-scientists

9. Santo K, Richtering SS, Chalmers J et al. Mobile phone apps to improve medication adherence: a systematic stepwise process to identify highquality apps. JMIR. Mhealth. Uhealth. 4(4), e132 (2016).

10. Haase J, Farris KB, Dorsch MP. Mobile Applications to improve medication adherence. Telemed. J. e-Health. 23(2), 75-79 (2017).

11. El Miedany Y, El Gaafary M, Youssef S et al. Toward electronic health recording: evaluation of electronic patient-reported outcome measures system for remote monitoring of early rheumatoid arthritis. J. Rheumatol. 43(12), 2106-2112 (2016).

12. Costedoat-Chalumeau N, Tamirou F, Piette JC. Treatment adherence in systemic lupus erythematosus and rheumatoid arthritis: time to focus on this important issue. Rheumatology. 57(9), 1507-1509 (2018).

13. Wabe N, Lee A, Wechalekar M et al. Adherence to combination DMARD therapy and treatment outcomes in rheumatoid arthritis: a longitudinal study of new and existing DMARD users. Rheumatol. Int. 37(6), 897 (2017).

14. Free C, Phillips G, Watson L et al. The effectiveness of mobile-health technologies to improve health care service delivery processes: a systematic review and meta-analysis. PLoS Med. 10(1), 1001363 (2013).

15. Azavedo R, Sousa H, Monteiro et al. Future perspectives of Smartphone applications for rheumatic diseases self-management. Rheumatol. Int. 35(3), 419-431 (2015).

16. Subhi Y, Bube HS, Bojsen RS et al. Expert involvement and adherence to medical evidence in medical mobile phone apps: a systematic review. JMIR. Mhealth. Uhealth. 27(3), 79 (2015).

17. Huckvale K, Adomaciciute S, Prieto JT et al. Smartphone apps for calculating insulin dose: a systematic review. BMC. Medicine. 13(1), 106
(2015).

18. Ajzen I. The theory of planned behavior. Organizational Behavior and Human Decision Processes. 50(2), 179-211 (1991).

19. Davis SF, Ellsworth MA, Payne HE et al. Health Behavior Theory in Popular Calorie Counting Apps: A Content Analysis. JMIR. Mhealth. Uhealth. 4(1), e19 (2016).

20. Simpson CC, Mazzeo SE. Calorie counting and fitness tracking technology: Associations with eating disorder symptomatology. Eat. Behav. 26, 89-92 (2017).

21. Matcham F, Rayner L, Steer S et al. The prevalence of depression in rheumatoid arthritis: a systematic review and meta-analysis. Rheumatology (Oxford). 52(12), 2136-2148 (2013).

22. Gilbert A. Development and adaptation of Rheumabuddy for young people with JIA, their families and people with RA residing in the UK. Ann. Rheum. Dis. 77, 1880 (2018).

23. Ferwerda M, Beugen S, Burik A et al. What patients think about E-health: patients' perspective on internet-based cognitive behavioral treatment for patients with rheumatoid arthritis and psoriasis. Clin. Rheumatol. 32(6), 869-873 (2013).

24. Lorig KR, Ritter PL, Laurent DD et al. The internet-based arthritis self-management program: A one-year randomized trial for patients with arthritis or fibromyalgia. Arthritis. Rheum. 59(7), 1009-1017 (2008).

25. Allam A, Kostova Z, Nakamoto K et al. The effect of social support features and gamification on a web-based intervention for rheumatoid arthritis patients: randomized controlled trial. J. Med. Internet. Res. 17(1), e14 (2015).

26. Nishiguchi S, Ito $H$, Yamada $M$ et al. Selfassessment tool of disease activity of rheumatoid arthritis by using a smartphone application. Telemed. J. eHealth. 20(3), 235-240 (2014).

27. Ward MM. Rheumatology visit frequency and changes in functional disability and pain in patients with rheumatoid arthritis. J. Rheumatol. 24(1), 35-42 (1997).

28. Veer VDS, Austin L, Sanders C et al. FRI0175 Using smartphones to improve remote monitoring of rheumatoid arthritis: completeness of patients' symptom reports. Ann. Rheum. Dis. 76: 547 (2017)

29. Walker A, Mueller RB et al. Disease activity dynamics in rheumatoid arthritis: patients' self-assessment of disease activity via WebApp. Rheumatology (Oxford). 56(10), 1707-1712 (2017).

30. Boulos KNM, Wheeler S, Tavares C et al. How smartphones are changing the face of mobile 
and participatory healthcare: an overview, with example from eCAALYX. Biomed. Eng. Online. 10(1), 24 (2011).

31. Proudfoot J, Clarke J, Birch MR et al. Impact of a mobile phone and web program on symptom and functional outcomes for people with mildto-moderate depression, anxiety and stress: a randomised controlled trial. BMC. Psychiatry. 13(1), 312 (2013).

32. DiFilippo KN, Huang WH, Andrade JE et al. The use of mobile apps to improve nutrition outcomes: a systematic literature review. J. Telemed. Telecare. 21(5), 243-253 (2015).

33. Nes AA, van Dulmen S, Eide E et al. The development and feasibility of a web-based intervention with diaries and situational feedback via smartphone to support self-management in patients with diabetes type 2. Diabetes. Res. Clin. Pract. 97(3), 385-393 (2012).

34. Krall R. The state of clinical research in the United States: an overview. Transforming Clinical Research in the United States: Challenges and Opportunities: Workshop Summary. (2010).

35. Khan A, Madden J. Mobile Devices as a resource in fathering health data: the role of mobile devices in the improvement of global health. International Conference on Computational Science and Computational Intelligence. (2016).

36. Yamada M, Aoyama T, Mori S et al. Objective assessment of abnormal gait in patients with rheumatoid arthritis using a smartphone. Rheumatol. Int. 32(12), 3869-3874 (2012).

37. Espinoza F, Blay LP, Coulon D et al. Handgrip strength measured by a dynamometer connected to a smartphone: a new applied health technology solution for the self-assessment of rheumatoid arthritis disease activity. Rheumatology. 55(5), 897-901 (2016).

38. Reade S, Sergeant JC, Sperrin M et al. FRI0590 Feasibility study of smartphone data collection for cloudy with a chance of pain: sustained engagement for daily self-reporting of disease severity in rheumatoid arthritis over two months. Arthritis. Rheumatol. 67: 10 (2015).

39. Kurniawan S. Older people and mobile phones: A multi-method investigation. Int. J. HumanComputer Studies. 66(12), 889-901 (2008).

40. Bol N, Helberger N, Weert JCM. Differences in mobile health app use: A source of new digital inequalities? The Information Society 34(3),183193 (2018).

41. Vaart R, Drossaert C, Heus M et al. Measuring actual eHealth literacy among patients with rheumatic diseases: a qualitative analysis of problems encountered using Health 1.0 and Health 2.0 applications. J. Med. Internet. Res. 15(2), e27 (2013).

42. Rahmati A, Tossell C, Shepard C et al. Exploring iPhone usage: the influence of socioeconomic differences on smartphone adoption, usage and usability. In Proceedings of the 14th international conference on Human-computer interaction with mobile devices and services (2012).

43. Yun H, Yang S, Nowell WB et al. Methotrexate use and fatigue in rheumatoid arthritis patients: results from a national patient registry. $A C R$. Abstract. 69(Suppl 10), (2017).

44. https://ec.europa.eu/digital-single-market/en/ news/commission-staff-working-documentexisting-eu-legal-framework-applicable-lifestyle 